\title{
The Evidence for Geary's Theory on the Role of Mitochondrial Functioning in Human Intelligence Is Not Entirely Convincing
}

\author{
Anna-Lena Schubert * (D) and Dirk Hagemann \\ Institute of Psychology, Heidelberg University, Hauptstr. 47-51, 69117 Heidelberg, Germany; \\ dirk.hagemann@psychologie.uni-heidelberg.de \\ * Correspondence: anna-lena.schubert@psychologie.uni-heidelberg.de
}

Received: 26 March 2020; Accepted: 16 July 2020; Published: 20 July 2020

check for updates

\begin{abstract}
Geary $(2018,2019)$ suggested that heritable and environmentally caused differences in mitochondrial functioning affect the integrity and efficiency of neurons and supporting glia cells and may thus contribute to individual differences in higher-order cognitive functioning and physical health. In our comment, we want to pose three questions aimed at different aspects of Geary's theory that critically evaluate his theory in the light of evidence from neurocognitive, cognitive enhancement, and behavioral genetics research. We question (1) if Geary's theory explains why certain cognitive processes show a stronger age-related decline than others; (2) if intervention studies in healthy younger adults support the claim that variation in mitochondrial functioning underlies variation in human intelligence; and (3) if predictions arising from the matrilineal heredity of mitochondrial DNA are supported by behavioral genetics research. We come to the conclusion that there are likely many more biological and social factors contributing to variation in human intelligence than mitochondrial functioning.
\end{abstract}

Keywords: general intelligence; mitochondrial functioning; behavioral genetics; cognitive enhancement; cognitive processes

\section{Introduction}

In the summary of his theory on intelligence differences, Geary (2019) outlined the elementary ideas of his hypothesis that individual differences in mitochondrial functioning are the main driving force for individual differences in human intelligence (Geary 2018, 2019). Geary suggested that heritable and environmentally caused differences in mitochondrial functioning affect the integrity and efficiency of neurons and supporting glia cells and may thus contribute to individual differences in higher-order cognitive functioning and physical health. His theory aimed to account for different phenomena of intelligence research, including the positive manifold (Spearman 1904), the association between intelligence and health (Batty et al. 2007; Deary 2008; Der et al. 2009) ${ }^{1}$, the joint age-related decline in performance across different cognitive domains (Rhemtulla and Tucker-Drob 2011; Salthouse 2009; Salthouse and Ferrer-Caja 2003; Tucker-Drob 2011; Tucker-Drob et al. 2014), and the greater variability in intelligence test scores in males than in females (Hedges and Nowell 1995; Johnson et al. 2008; Wai et al. 2010). As such, Geary's theory is very compelling because it provides an elegant account of many important empirical phenomena of intelligence research. Moreover, it integrates biological and

1 We thank an anonymous reviewer for pointing out that recent evidence suggests that this association may reverse in highly intelligent individuals (i.e., those within the upper $2 \%$ of the general population), who may be at a higher risk than the general population to develop certain psychological disorders as well as physiological conditions (Karpinski et al. 2018). 
cognitive approaches to studying intelligence by acknowledging that elementary cognitive processes underlying human intelligence are bound to the integrity of the brain, which is one of many bodily organs and, therefore, affected by basic physiology.

In our comment, we want to pose three questions aimed at different aspects of Geary's theory that critically evaluate his theory in the light of evidence from neurocognitive, cognitive enhancement, and behavioral genetics research.

\subsection{Can Geary's Theory Explain Why Certain Cognitive Processes Show a Stronger Age-Related Decline Than Others?}

Geary (2018) argued that parameters of certain cognitive processes such as working memory (WM), which "refers to a system, or set of processes, holding mental representations temporarily available for use in thought and action" (Oberauer et al. 2018, p. 886), are more strongly related to intelligence and show greater age-related decline than parameters of other cognitive processes, such as short-term memory (STM), due to higher energy demands. Because more energy-demanding systems show greater metabolic activity than less energy-demanding systems, they create more reactive oxygen, which results in more rapid accumulation of mutations across the life span.

This argument relies on the assumption that working memory processes require intermodular neural connections that are supported by intact axonal connections between modules, whereas other cognitive processes rely to a larger degree on intramodular connections. Hence, both higher energy use and the dependence on a larger number of intermodular axonal connections are supposed to account for the greater age-related decline in WM than in STM. We believe that this conclusion is neither supported by cognitive theories on memory systems nor by neuroscientific evidence. Cognitive psychologists have been abandoning a strict distinction between WM and STM towards a broader definition of working memory that includes short-term memory processes (Oberauer et al. 2018). If there is a conceptual distinction between the two memory systems, it typically entails that STM involves the mere maintenance of information, whereas WM involves the simultaneous maintenance and manipulation of information (Cowan 2017). This additional manipulation of information may indeed require higher levels of cellular energy. However, there is ample evidence that the encoding, maintenance, and retrieval of information required both in WM and STM rely on long-range intermodular neural connections. In particular, these processes have been shown to require the integration of neural activity elicited from the ventrolateral prefrontal cortex, the inferior temporal cortex, the ventral posterior parietal cortex, and the medial temporal lobe (Nee and Jonides 2011, 2013a, 2013b; Öztekin et al. 2008). Therefore, it is implausible to assume that individual differences in axonal integrity and neural plasticity or age-related myelin degradation account for greater age-related decline in WM than in STM, as both WM and STM processes require long-distance intermodular information transmission.

In addition, language-related abilities such as verbal understanding and verbal production, which are reflected in measures of crystallized intelligence, stay relatively stable across the lifespan and show a much more decelerated age-related decline than both WM and STM (Horn and Cattell 1967; Salthouse 2004). However, the same processes are known to heavily rely on intermodular connections (Barbey 2018; Silbert et al. 2014). As such, the discrepancy in age-related trajectories of fluid and crystallized intelligence also contradicts the idea that networks relying on long-distance intermodular information transmission show a greater age-related decline due to more extensive myelin degradation.

\subsection{Do Intervention Studies in Healthy Younger Adults Support the Claim That Variation in Mitochondrial Functioning Underlies Variation in Human Intelligence?}

One very elegant test of Geary's hypothesis would be to increase mitochondrial functioning through pharmaceutical or training-based interventions and to assess whether these interventions show effects on general cognitive performance. Geary (2018) cited several studies in which nutritional supplementation using ketone agents, creatine, coenzyme Q10, or resveratrol, which affect different 
aspects of mitochondrial functioning, led to increases in cognitive performance in older adults and in adults with neurodegenerative diseases, such as Parkinson's and Alzheimer's disease (Henderson et al. 2009; Li et al. 2015; Witte et al. 2014). However, these effects seem to only hold for older individuals or individuals with certain neurodegenerative diseases, while evidence for cognitive enhancement by nutritional supplementation in younger adults is limited.

A recent review on the beneficial effects of creatine supplementation summarized three studies assessing the effects on intelligence test performance in younger adults (Avgerinos et al. 2018). Creatine supplies energy to cells with increased energy demands, and its higher-energy phosphate bonds can be used for immediate ATP replenishment in energy-demanding situations, such as cognitive tasks, that require high neural energy use (Persky and Brazeau 2001). However, the studies summarized by Avgerinos et al. (2018) do not provide compelling evidence that increased creatine availability increases cognitive functioning in healthy young adults. One of these studies found no effect of creatine supplementation on cognitive performance (Rawson et al. 2008), while another found a large effect in comparison to a placebo condition that could, however, be attributed to substantial baseline differences in intelligence between the two groups (Ling et al. 2009). The only study that reported compelling evidence for enhanced cognitive performance following creatine supplementation contained a sample of vegetarians (Rae et al. 2003), who typically show lower plasma creatine levels due to their diet (Delanghe et al. 1989).

Similarly, effects of resveratrol administration seem to protect against neurodegenerative effects on cognitive function (Foti Cuzzola et al. 2011; Sun et al. 2010; Witte et al. 2014). Resveratrol is best known as one of several wine polyphenols thought to be responsible for the health benefits of moderate regular wine consumption (Keylor et al. 2015). It is synthesized by plants undergoing infectious or ionizing radiation and acts as a potent antioxidant (Malhotra et al. 2015; Salehi et al. 2018). However, despite showing promising effects in the treatment of neurodegenerative diseases, several studies failed to observe any benefits of resveratrol administration on cognitive functioning in healthy young adults despite observing resveratrol-related increases in cerebral blood flow (Kennedy et al. 2010; Wightman et al. 2014, 2015; Wong et al. 2013).

Taken together, these results suggest that these nutritional supplementations only affect cognitive functioning in older adults, in individuals with certain neurodegenerative diseases, and in individuals with dietary restrictions. In a similar vein, animal studies have shown that experimental interventions modulating mitochondrial fusion/fission dynamics can facilitate neural regeneration (Chien et al. 2018), underlining the role of mitochondrial dynamics in degenerative neurocognitive processes. Hence, these studies cannot be cited to support Geary (2018) hypothesis that individual differences in mitochondrial functioning underlie natural variation in intelligence; instead, they only lend credence to the idea that age-related cognitive decline may be mediated by impairments in mitochondrial functioning.

\subsection{Are Predictions Arising from the Matrilineal Heredity of Mitochondrial DNA Supported by Behavioral Genetics Research?}

There is evidence that variations in mitochondrial DNA (mtDNA) have an effect on mitochondrial functioning and several body systems, including the brain (Geary 2018, p. 1034). When, in turn, differences in mitochondrial functioning have an effect on general intelligence, then one would expect that differences in mtDNA are also associated with differences in intelligence. In particular, individuals with similar mtDNA would be expected to show rather similar levels of intelligence, and individuals with dissimilar mtDNA would be expected to show rather dissimilar levels of intelligence. Because mtDNA has a matrilineal heredity, it follows that the mtDNA of a mother and her offspring is much more similar (identical devoid of mutations) than in the case of a father and his offspring. In short, one may conclude that the mother-offspring correlation of intelligence must be larger than the father-offspring correlation of intelligence and that the size of this difference gives a clue on the magnitude of how much mtDNA differences would drive differences in intelligence. However, empirical data do not support this prediction. Whitley et al. (2011) analyzed data from the 1958 cohort of the National Child 
Development Study, including offspring data from the children of the original study members. They reported that the mother-offspring correlation of IQ scores was 0.30 , and that the father-offspring correlation was 0.31 (these findings are based on 2202 parent-offspring pairs). Thus, there was no larger correlation for mothers than for fathers, which suggests that the similarity/dissimilarity of mtDNA does not affect the similarity/dissimilarity of IQ scores.

A tentative explanation of this null finding may be that there are no main effects of mtDNA variations on mitochondrial functioning, but that the latter is affected by mito-nuclear interactions only. However, this argument is at odds with the evidence that differences in mtDNA do have sizable effects on mitochondrial and body system functioning, as noted above. Another tentative explanation of this null finding may be that differences in mitochondrial functioning translate only with a very small effect size into differences in intelligence - too small to be detected in the study of Whitley et al. (2011). In this case, there is a place for many more factors that contribute to the variance of intelligence, which run outside the mechanisms of mitochondrial functioning, be they biological or social in nature.

\section{Conclusions}

Taken together, Geary's theory is very compelling because it provides an elegant account of many important empirical phenomena of intelligence research. Moreover, it integrates biological and cognitive approaches to studying intelligence by acknowledging that elementary cognitive processes underlying human intelligence are bound to the integrity of the brain, which is one of many bodily organs and is, therefore, affected by basic physiology. We discussed different findings from neurocognitive, cognitive enhancement, and behavioral genetics research that challenged various aspects of his theory. First, we discussed recent research from cognitive psychology and cognitive neuroscience that questioned Geary's prediction that networks relying on long-distance intermodular information transmission show a greater age-related decline due to more extensive myelin degradation. As both WM and STM processes require long-distance intermodular information transmission, it is implausible to assume that individual differences in axonal integrity and neural plasticity or age-related myelin degradation account for the phenomenon of greater age-related decline in WM than in STM. Second, we looked into the nutritional interventions discussed by Geary and concluded that these nutritional supplementations only affect cognitive functioning in older adults, in individuals with certain neurodegenerative diseases, and in individuals with dietary restrictions, whereas they do not show any cognitive benefits in healthy young adults. Third, we reviewed evidence from behavioral genetics research that challenged the prediction arising from the matrilineal heredity of mitochondrial DNA that the mother-offspring correlation of intelligence should be larger than father-offspring correlation. Data from a large-scale national cohort study showed that mother- and father-offspring correlations were virtually identical.

While our reservations based on neurocognitive and cognitive enhancement research only concerned certain parts of Geary's theory, the discussed evidence from behavioral genetics research questioned a core assumption of the theory, namely that variations in mitochondrial DNA have an effect on mitochondrial functioning, which, in turn, has an effect on human intelligence. While we cannot and do not want to rule out that some amount of variation in human intelligence can be attributed to individual differences in mitochondrial functioning, the findings discussed above let us conclude that there are likely many more factors contributing to individual differences in intelligence, ranging from genes (e.g., genome-wide polygenic scores explain up to $10 \%$ of variance in intelligence; (Plomin and Stumm 2018)) to structural (e.g., white-matter tract integrity in the forceps minor, the corticospinal tract, the anterior thalamic radiation, the right superior longitudinal fasciculus, the uncinate fasciculus, the rostrolateral prefrontal cortex, and the inferior parietal lobe; (Booth et al. 2013; Pineda-Pardo et al. 2016; Kievit et al. 2016; Tamnes et al. 2010; Wendelken et al. 2017)) and functional brain characteristics (e.g., activation of fronto-parietal brain networks and functional connectivity related to higher-order cognitive processes; (Basten et al. 2015; Jung and Haier 2007; Hilger et al. 2017; Schubert et al. 2020)), mediating cognitive processes (e.g., processing speed, attentional control, working memory; (Engle 
2018; Kovacs and Conway 2016; Schubert and Frischkorn 2020)), environmental influences (e.g., prenatally available polyunsaturated fatty acids; (Cohen et al. 2005; Lassek and Gaulin 2008)), and developmental interdependencies (Van Der Maas et al. 2006).

Author Contributions: Conceptualization, A.-L.S. and D.H.; writing-original draft preparation, A.-L.S. and D.H.; writing-review and editing, A.-L.S. and D.H.; visualization. All authors have read and agreed to the published version of the manuscript.

Funding: This research received no external funding.

Conflicts of Interest: The authors declare no conflict of interest.

\section{References}

Avgerinos, Konstantinos I., Nikolaos Spyrou, Konstantinos I. Bougioukas, and Dimitrios Kapogiannis. 2018. Effects of creatine supplementation on cognitive function of healthy individuals: A systematic review of randomized controlled trials. Experimental Gerontology 108: 166-73. [CrossRef]

Barbey, Aron K. 2018. Network neuroscience theory of human intelligence. Trends in Cognitive Sciences 22: 8-20. [CrossRef]

Basten, Ulrike, Kirsten Hilger, and Christian J. Fiebach. 2015. Where smart brains are different: A quantitative meta-analysis of functional and structural brain imaging studies on intelligence. Intelligence 51: 10-27. [CrossRef]

Batty, G. David, Ian J. Deary, and Linda S. Gottfredson. 2007. Premorbid (Early Life) IQ and later mortality risk: Systematic review. Annals of Epidemiology 17: 278-88. [CrossRef] [PubMed]

Booth, Tom, Mark E. Bastin, Lars Penke, Susana Muñoz Maniega, Catherine Murray, Natalie A. Royle, Alan J. Gow, Janie Corley, Ross D. Henderson, Maria del C. Valdés Hernández, and et al. 2013. Brain white matter tract integrity and cognitive abilities in community-dwelling older people: The Lothian birth cohort, 1936. Neuropsychology 27: 595-607. [CrossRef]

Chien, Ling, Min-Zong Liang, Chu-Yuan Chang, Chen Wang, and Linyi Chen. 2018. Mitochondrial therapy promotes regeneration of injured hippocampal neurons. Biochimica et Biophysica Acta (BBA)-Molecular Basis of Disease 1864, Pt B: 3001-12. [CrossRef] [PubMed]

Cohen, Joshua T., David C. Bellinger, William E. Connor, and Bennett A. Shaywitz. 2005. A quantitative analysis of prenatal intake of N-3 polyunsaturated fatty acids and cognitive development. American Journal of Preventive Medicine 29: 366.e12. [CrossRef] [PubMed]

Cowan, Nelson. 2017. The many faces of working memory and short-term storage. Psychonomic Bulletin E Review 24: 1158-70. [CrossRef]

Deary, Ian. 2008. Why do intelligent people live longer? Nature 456: 175-76. [CrossRef]

Delanghe, Joris R., Jean-Paul De Slypere, Marc L. De Buyzere, Johan Robbrecht, Roger Wieme, and Alex Vermeulen. 1989. Normal reference values for creatine, creatinine, and carnitine are lower in vegetarians. Clinical Chemistry 35: 1802-3. [CrossRef]

Der, Geoff, G. David Batty, and Ian J. Deary. 2009. The association between IQ in adolescence and a range of health outcomes at 40 in the 1979 US national longitudinal study of youth. Intelligence, Intelligence, Health and Death: The Emerging Field of Cognitive Epidemiology 37: 573-80. [CrossRef] [PubMed]

Engle, Randall W. 2018. Working memory and executive attention: A revisit. Perspectives on Psychological Science: A Journal of the Association for Psychological Science 13: 190-93. [CrossRef]

Foti Cuzzola, V., Rosella Ciurleo, Sabrina Giacoppo, Silvia Marino, and Placido Bramanti. 2011. Role of resveratrol and its analogues in the treatment of neurodegenerative diseases: Focus on recent discoveries. CNS $\mathcal{E}$ Neurological Disorders Drug Targets 10: 849-62. [CrossRef]

Geary, David C. 2018. Efficiency of mitochondrial functioning as the fundamental biological mechanism of general intelligence (g). Psychological Review 125: 1028-50. [CrossRef]

Geary, David C. 2019. Mitochondria as the linchpin of general intelligence and the link between $g$, health, and aging. Journal of Intelligence 7: 25. [CrossRef] [PubMed]

Hedges, Larry V., and Amy Nowell. 1995. Sex differences in mental test scores, variability, and numbers of high-scoring individuals. Science 269: 41-45. [CrossRef] 
Henderson, Samuel T., Janet L. Vogel, Linda J. Barr, Fiona Garvin, Julie J. Jones, and Lauren C. Costantini. 2009. Study of the Ketogenic agent AC-1202 in mild to moderate Alzheimer's Disease: A randomized, double-blind, placebo-controlled, multicenter trial. Nutrition \& Metabolism 6: 31. [CrossRef]

Hilger, Kirsten, Matthias Ekman, Christian J. Fiebach, and Ulrike Basten. 2017. Efficient hubs in the intelligent brain: Nodal efficiency of hub regions in the salience network is associated with general intelligence. Intelligence 60: 10-25. [CrossRef]

Horn, John L., and Raymond B. Cattell. 1967. Age differences in fluid and crystallized intelligence. Acta Psychologica 26: 107-29. [CrossRef]

Johnson, Wendy, Andrew Carothers, and Ian J. Deary. 2008. Sex differences in variability in general intelligence: A new look at the old question. Perspectives on Psychological Science 3: 518-31. [CrossRef]

Jung, Rex E., and Richard J. Haier. 2007. The Parieto-Frontal Integration Theory (P-FIT) of intelligence: Converging neuroimaging evidence. The Behavioral and Brain Sciences 30: 135-54; discussion 154-87. [CrossRef] [PubMed]

Karpinski, Ruth I., Audrey M. Kinase Kolb, Nicole A. Tetreault, and Thomas B. Borowski. 2018. High intelligence: A risk factor for psychological and physiological Overexcitabilities. Intelligence 66: 8-23. [CrossRef]

Kennedy, David O., Emma L. Wightman, Jonathon L. Reay, Georg Lietz, Edward J. Okello, Anthea Wilde, and Crystal F. Haskell. 2010. Effects of resveratrol on cerebral blood flow variables and cognitive performance in humans: A double-blind, placebo-controlled, crossover investigation. The American Journal of Clinical Nutrition 91: 1590-97. [CrossRef] [PubMed]

Keylor, MitchellH, Bryan S. Matsuura, and Corey R. J. Stephenson. 2015. Chemistry and biology of resveratrol-derived natural products. Chemical Reviews 115: 8976-9027. [CrossRef] [PubMed]

Kievit, Rogier A., Simon W. Davis, John Griffiths, Marta M. Correia, Cam-CAN, and Richard N. Henson. 2016. A watershed model of individual differences in fluid intelligence. Neuropsychologia 91: 186-98. [CrossRef]

Kovacs, Kristof, and Andrew R. A. Conway. 2016. Process overlap theory: A unified account of the general factor of intelligence. Psychological Inquiry 27: 151-77. [CrossRef]

Lassek, William D., and Steven J. C. Gaulin. 2008. Waist-Hip ratio and cognitive ability: Is gluteofemoral fat a privileged store of neurodevelopmental resources? Evolution and Human Behavior 29: 26-34. [CrossRef]

Li, Zhenguang, Pengfei Wang, Zhancai Yu, Yannan Cong, Hairong Sun, Jiangshan Zhang, Jinbiao Zhang, Chao Sun, Yong Zhang, and Xiaohua Ju. 2015. The effect of creatine and coenzyme q10 combination therapy on mild cognitive impairment in Parkinson's Disease. European Neurology 73: 205-11. [CrossRef]

Ling, Jonathan, Minos Kritikos, and Brian Tiplady. 2009. Cognitive effects of Creatine Ethyl Ester supplementation. Behavioural Pharmacology 20: 673-79. [CrossRef] [PubMed]

Malhotra, Ashim, Sundeep Bath, and Fawzy Elbarbry. 2015. An Organ System Approach to Explore the Antioxidative, Anti-Inflammatory, and Cytoprotective Actions of Resveratrol. Review Article. Oxidative Medicine and Cellular Longevity. London: Hindawi. [CrossRef]

Nee, Derek Evan, and John Jonides. 2011. Dissociable contributions of prefrontal cortex and the hippocampus to short-term memory: Evidence for a 3-State model of memory. NeuroImage 54: 1540-48. [CrossRef]

Nee, Derek Evan, and John Jonides. 2013a. Trisecting representational states in short-term memory. Frontiers in Human Neuroscience 7: 796. [CrossRef] [PubMed]

Nee, Derek Evan, and John Jonides. 2013b. Neural Evidence for a 3-State model of visual short-term memory. NeuroImage 74: 1-11. [CrossRef] [PubMed]

Oberauer, Klaus, Stephan Lewandowsky, Edward Awh, Gordon D. A. Brown, Andrew Conway, Nelson Cowan, Christopher Donkin, Simon Farrell, Graham J. Hitch, Mark J. Hurlstone, and et al. 2018. Benchmarks for models of short-term and working memory. Psychological Bulletin 144: 885-958. [CrossRef]

Öztekin, Ilke, Brian McElree, Bernhard P. Staresina, and Lila Davachi. 2008. Working memory retrieval: Contributions of the left prefrontal cortex, the left posterior parietal cortex, and the hippocampus. Journal of Cognitive Neuroscience 21: 581-93. [CrossRef] [PubMed]

Persky, Adam M., and Gayle A. Brazeau. 2001. Clinical pharmacology of the dietary supplement Creatine monohydrate. Pharmacological Reviews 53: 161-76. [PubMed]

Pineda-Pardo, José Angel, Kenia Martínez, Francisco J. Román, and Roberto Colom. 2016. Structural efficiency within a parieto-frontal network and cognitive differences. Intelligence 54: 105-16. [CrossRef]

Plomin, Robert, and Sophie von Stumm. 2018. The new genetics of intelligence. Nature Reviews Genetics 19: 148-59. [CrossRef] 
Rae, Caroline, Alison L. Digney, Sally R. McEwan, and Timothy C. Bates. 2003. Oral Creatine monohydrate supplementation improves Brain performance: A double-blind, placebo-controlled, cross-over trial. Proceedings. Biological Sciences 270: 2147-50. [CrossRef]

Rawson, Eric S., Harris R. Lieberman, Talia M. Walsh, Sylwia M. Zuber, Jaclyn M. Harhart, and Tracy C. Matthews. 2008. Creatine supplementation does not improve cognitive function in young adults. Physiology $\mathcal{E}$ Behavior 95: 130-34. [CrossRef]

Rhemtulla, Mijke, and Elliot M. Tucker-Drob. 2011. Correlated longitudinal changes across linguistic, achievement, and psychomotor domains in early childhood: Evidence for a global dimension of development. Developmental Science 14: 1245-54. [CrossRef]

Salehi, Bahare, Abhay Prakash Mishra, Manisha Nigam, Bilge Sener, Mehtap Kilic, Mehdi Sharifi-Rad, Patrick Valere Tsouh Fokou, Natália Martins, and Javad Sharifi-Rad. 2018. Resveratrol: A double-edged sword in health benefits. Biomedicines 6. [CrossRef]

Salthouse, Timothy A. 2004. Localizing age-related individual differences in a hierarchical structure. Intelligence 32: 541-61. [CrossRef]

Salthouse, Timothy A. 2009. When does age-related cognitive decline begin? Neurobiology of Aging 30: 507-14. [CrossRef] [PubMed]

Salthouse, Timothy A., and Emilio Ferrer-Caja. 2003. What needs to be explained to account for age-related effects on multiple cognitive variables? Psychology and Aging 18: 91-110. [CrossRef] [PubMed]

Schubert, Anna-Lena, and Gidon T. Frischkorn. 2020. Neurocognitive psychometrics of intelligence: How measurement advancements unveiled the role of mental speed in intelligence differences. Current Directions in Psychological Science 29: 140-46. [CrossRef]

Schubert, Anna-Lena, Dirk Hagemann, Christoph Löffler, Jan Rummel, and Stefan Arnau. 2020. A chronometric model of the relationship between frontal midline theta functional connectivity and human intelligence. Journal of Experimental Psychology: General. [CrossRef]

Silbert, Lauren J., Christopher J. Honey, Erez Simony, David Poeppel, and Uri Hasson. 2014. Coupled neural systems underlie the production and comprehension of naturalistic narrative speech. Proceedings of the National Academy of Sciences of the United States of America 111: E4687-E4696. [CrossRef]

Spearman, C. 1904. 'General Intelligence,' objectively determined and measured. The American Journal of Psychology 15: 201-93. [CrossRef]

Sun, Albert Y., Qun Wang, Agnes Simonyi, and Grace Y. Sun. 2010. Resveratrol as a therapeutic agent for neurodegenerative diseases. Molecular Neurobiology 41: 375-83. [CrossRef]

Tamnes, Christian K., Ylva Østby, Kristine B. Walhovd, Lars T. Westlye, Paulina Due-Tønnessen, and Anders M. Fjell. 2010. Intellectual abilities and white matter microstructure in development: A diffusion tensor imaging study. Human Brain Mapping 31: 1609-25. [CrossRef]

Tucker-Drob, Elliot M. 2011. Global and domain-specific changes in cognition throughout adulthood. Developmental Psychology 47: 331-43. [CrossRef] [PubMed]

Tucker-Drob, Elliot M., Daniel A. Briley, John M. Starr, and Ian J. Deary. 2014. Structure and correlates of cognitive aging in a narrow age cohort. Psychology and Aging 29: 236-49. [CrossRef]

Van Der Maas, Han L. J., Conor V. Dolan, Raoul P. P. P. Grasman, Jelte M. Wicherts, Hilde M. Huizenga, and Maartje E. J. Raijmakers. 2006. A dynamical model of general intelligence: The positive manifold of intelligence by mutualism. Psychological Review 113: 842-61. [CrossRef]

Wai, Jonathan, Megan Cacchio, Martha Putallaz, and Matthew C. Makel. 2010. Sex differences in the right tail of cognitive abilities: A 30 year examination. Intelligence 38: 412-23. [CrossRef]

Wendelken, Carter, Emilio Ferrer, Simona Ghetti, Stephen K. Bailey, Laurie Cutting, and Silvia A. Bunge. 2017. Frontoparietal structural connectivity in childhood predicts development of functional connectivity and reasoning ability: A large-scale longitudinal investigation. The Journal of Neuroscience: The Official Journal of the Society for Neuroscience 37: 8549-58. [CrossRef]

Whitley, Elise, Catharine R. Gale, Ian J. Deary, Mika Kivimaki, and G. David Batty. 2011. Association of maternal and paternal IQ with offspring conduct, emotional, and attention problem scores. Transgenerational evidence from the 1958 British birth cohort study. Archives of General Psychiatry 68: 1032-38. [CrossRef] [PubMed] 
Wightman, Emma L., Jonathon L. Reay, Crystal F. Haskell, Gary Williamson, Tristan P. Dew, and David O. Kennedy. 2014. Effects of resveratrol alone or in combination with piperine on cerebral blood flow parameters and cognitive performance in human subjects: A Randomised, double-blind, placebo-controlled, cross-over investigation. The British Journal of Nutrition 112: 203-13. [CrossRef]

Wightman, Emma L., Crystal F. Haskell-Ramsay, Jonathon L. Reay, Gary Williamson, Tristan Dew, Wei Zhang, and David O. Kennedy. 2015. The effects of chronic trans-resveratrol supplementation on aspects of cognitive function, mood, sleep, health and cerebral blood flow in healthy, young humans. British Journal of Nutrition 114: 1427-37. [CrossRef]

Witte, A. Veronica, Lucia Kerti, Daniel S. Margulies, and Agnes Flöel. 2014. Effects of resveratrol on memory performance, hippocampal functional connectivity, and glucose metabolism in healthy older adults. Journal of Neuroscience 34: 7862-70. [CrossRef]

Wong, Rachel H. X., Narelle M. Berry, Alison M. Coates, Jonathan D. Buckley, Janet Bryan, Iris Kunz, and Peter R. C. Howe. 2013. Chronic resveratrol consumption improves brachial flow-mediated dilatation in healthy obese adults. Journal of Hypertension 31: 1819-27. [CrossRef]

(C) 2020 by the authors. Licensee MDPI, Basel, Switzerland. This article is an open access article distributed under the terms and conditions of the Creative Commons Attribution (CC BY) license (http://creativecommons.org/licenses/by/4.0/). 